\title{
Geografía y salud: \\ temas y perspectivas en América Latina
}

\author{
Geography and health: \\ themes and perspectives in Latin America
}

Luisa Iñiguez Rojas 1

\footnotetext{
1 Centro de Estudios de Salud y Bienestar Humano. Universidad de La Habana. San Lázaro y L.

Município Plaza de la Revolución, Ciudad de la Habana, Cuba. cesbh@comuhuh.cu
}

Abstract Relations between geography and health have been recognized since ancient times. Investigation of such relations has been characterized by isolated and scant efforts. This article aims to explore potential links between geography's theoretical and methodological frame of reference and knowledge and interpretation of the population's health. It approaches the antecedents of medical or health-rel ated geography and the use of the theoretical and methodological framework of geographic space, as well as identifying alternatives for its implementation. Finally, it raises several points relating to current devel opment in the relations between geography and health in Latin America, as well as alternatives for such development.

Key words Medical Geography; Geographical Space; Spatial Analysis; Health

Resumen Las relaciones entre la geografía y la salud fueron reconocidas desde la Antiguedad. Esfuerzos aislados e inconstantes caracterizan la investigación de estas relaciones. El presente artículo tiene como objetivo una exploración de las potencialidades de articulación del referencial teórico y metodológi co de la geografía en el conocimiento y la interpretación de la salud de la población. Aborda los antecedentes de la geografía médica o dela salud, la utilización del marco teórico-metodológi co del espacio geográfico, e identifica las al ternativas para su operacionalización. Por último son colocadas algunas reflexiones acerca del desarrollo actual de las relaciones entre la geografía y la salud en América Latina, así como las alternativas de su desarrollo.

Palabras clave Geografía Médica; Espacio Geográfico; Análisis Espacial; Salud 


\section{Introducción}

A pesar de que aún asombra en algunos contextos institucionales, la relación entre las Ciencias Geográficas y las Ciencias de la Salud, existe una amplia documentación que argumenta esta articulación desde los albores del saber humano. Durante la más primaria interacción hombre-naturaleza y procurando esencialmente subsistir, los hombres fueron creando los mecanismos para identificar lo sano y lo perjudicial.

Ocupada siempre en la distribución de fenómenos y procesos, la geografía, a pesar de los esfuerzos aislados e inconstantes, no ha logrado incorporar la lectura del estado biopsicosocial del hombre como una de sus preocupaciones centrales, en los distintos contextos regionales y nacionales donde ha evolucionado y particularmente en Latinoamerica.

Las causas iniciales pudieran asociarse a que inmersa durante largas décadas en la dicotomía entre lo natural y lo social o económico, entre los “ismos" de la determinación, la posibilidad y la necesidad, entre la cuantificación y la cualificación, se debatió y aún hoy se debate en la búsqueda de la justa medida para el conocimiento de lo humano y lo social.

¿Dónde y cómo se inscribían los grupos humanos, en la larga y profusa historia de estudios geográficos regionales, urbanos, agrarios y otros?. ¿Conseguían identificar los problemas e inquietudes de estos grupos?.

Relacionado con estas interrogantes el geógrafo francés Paul Claval (1987) planteara que las observaciones que sus profesores hacían de sus disertaciones y memorias, coincidían con las que él hiciese después como catedrático "no se ven los hombres y faltan los instrumentos para aprehenderlos y articular los modelos sociales a los modelos del hombre"(Claval, 1987:242).

Una conmovedora sentencia del eminente geógrafo brasileño Milton Santos reafirma estos hechos: - “Destemporalizando el espacio y deshumanizándolo, la geografía acabó por darle la espalda a su objeto y siendo una viuda del espacio" (Santos, 1990:107).

Los antecedentes del tema y la geografía médica o de la salud

En la intensa fragmentación histórica de la Geografía aparecieron direcciones que de forma más o menos directa se acercaban al tema del bienestar. La Geografía Humana, de la Población, Urbana, Rural, Histórica, Social, Políti- ca y Cultural, se imbricaban en una borrosa trama con las surgidas o reforzadas en las últimas dos décadas tales como la del Comportamiento, del Género, de la Percepción, Humanística y Ambiental.

Sugerida inicialmente como una nueva organización de la Geografía Humana, en la década del 70 la Geografía del Bienestar, proponía un enfoque integrador capaz de borrar las fronteras innecesariamente incrementadas entre las disciplinas geográficas que abordaban el tema del bienestar humano (Smith, 1980).

Los antecedentes teóricos mas notables se reconocen en la Antropogeografía de Ratzel (1948) y la Geografía Humana de Vidal de la Blache (1922). M. Sorre depliega el potente arsenal de los conceptos de género de vida, de ecúmene y complejos patógenos, interpretando las enfermedades y las muertes como tema o aspecto central de la Geografía o Ecología Humana, trazándo fundamentos de la Geografía M édica aún vigentes, aunque poco explorados (Sorre, 1955).

Los términos Geografía Médica y Geografía de la Salud resultaron los mas ampliamente debatidos y ampliamente aceptados para identificar aquella dirección de la geografía, que surgida en el pasado siglo y calificada hoy “como una antigua perspectiva y una nueva especialización" (Meade et al., 1988:1) se ocupa de la aplicación del conocimiento geográfico, métodos y técnicas a la investigación en salud, en la perspectiva de la prevención de enfermedades (Verhasselt, 1993:482).

Otras denominaciones han sido adoptadas por países y regiones, entre las que se destacan con alguna connotación evolutiva, la Topografía M édica, Geografía de las patologías, de las enfermedades y las muertes, Geomedicina, Geoepidemiología y Ecología Médica. Según la convencional división de las ciencias que se adopte, se ha considerado como parte de la Geografía Humana (Sorre, 1955), de la Ecología Humana o Social., como una disciplina de borderline (Pyle, 1977), en la intersección entre la geografía, la medicina y la biología (Darchenkova, 1986) o entre las ciencias sociales, físicas y biológicas (Meade et al., 1988). Se consideran profesionales destacados en esta dirección geográfica Sorre; May; Stamp; Learmonth; Mc Glashan; Shoshin; Pyle; Momiyama-Sakamoto; Philips; Verhasselt, entre otros.

Frecuentemente se cuestiona la existencia independiente de la Geografía Médica. Al respecto el profesor brasilero L. J. da Silva considera que la Geografía Médica nunca se estableció firmemente como disciplina distinta a la Epidemiología (Silva, 1992). Esta afirmación 
que podemos aceptar, como opinión de epidemiológos y otros profesionales de las Ciencias Médicas, no excluye la incorporación del lenguaje de expresión geográfica (cartografía) por los epidemiólogos, ni las renovadas perspectivas que los conceptos y técnicas geográficas, han abierto a los profesionales de la salud. De forma similar, la investigación geográfica en salud, incorpora las técnicas bioestadísticas, ampliamente desarrolladas por los epidemiólogos y otros profesionales de la salud.

La importancia de la Geografía M édica, se concreta al constituirse en Lisboa la Comisión de Geografía Médica de la UGI en 1949 y en el informe que esta comisión presentara en 1952 en aras de impulsar su desarrollo. Cuarenta años despues, en el Congreso de la Unión Geográfica Internacional celebrado en Washington, se modifica el nombre de esta Comisión por el de Ambiente-Salud y desarrollo.

Sin embargo, a excepción de algunos países entre los que se destacan el Reino Unido, Francia, Bélgica, Estados Unidos, Alemania y Rusia entre otros, la Geografía M édica o de la Salud no ha conseguido consolidarse como dirección científica y es poco conocida o desconocida, hasta entre los profesionales de la geografía.

La Geografía Médica o de la Salud, frecuentemente se divide en dos principales campos de investigación : la Nosogeografía o Geografía Médica tradicional, encargada de la identificación y análisis de patrones de distribución espacial de enfermedades y la Geografía de la atención médica o de salud, ocupada en la distribución y planeamiento de componentes infraestructurales y de recursos humanos del Sistema de Atención Médica.

Una dirección mas holística y reciente, se desdoblaría en la aproximación a la diferenciación del bienestar, las condiciones y calidad de la vida incorporando, a los indicadores "clásicos", Ios referidos a la enfermedad o la muerte en espacios poblacionales, especial mente urbanos, o en los estudios de estado y situación de salud en diferentes unidades territoriales de países y regiones (Castellanos, 1992; Iñiguez, 1994).

Las dos primeras aunque en íntima relación, pueden considerarse de forma independiente. Numerosos estudios de distribución geográfica de la morbilidad o mortalidad, no se proponen la aplicación de los resultados a la gestión en salud. La tercera merece una especial reflexión. La Geografía no se relaciona con el bienestar y la salud de las poblaciones sólo en el contexto de la Geografía Médica. Mas indirecta y poco explorada, la información del proceso salud-enfermedad puede ser incorpo- rada siempre que se aborde la desigual distribución de componentes y procesos socio-económicos, incluyendo los socioculturales.

En cualquiera de estas direcciones la geografía se aproxima a la salud de las colectividades, de los entornos comunitarios. Su mas notable especificidad es privilegiar el espacio y su principal problema articular el ¿dónde? con los ¿quiénes?.

Discernir las relaciones entre la Geografía y la salud. Privilegiar el espacio geográfico

La geografía deviene frente a la salud, no un simple reservorio de climas, contaminantes, de microbios, de vectores de transmisión infecciosa, etc; sino un espacio históricamente estructurado, donde también se expresan las consecuencias benéficas y destructivas de la organización social (Breilh et al., 1988).

El espacio es frecuentemente utilizado, tanto por geógrafos como por epidemiólogos como sinónimo de área, superficie o lugar (Mausner \& Kramer, 1985; Sounis, 1985; Lilienfield \& Lilienfield, 1986; Jenicek \& Cléroux, 1987; Rouquayrol, 1994). Así la distribución espacial es una distribución geográfica, a partir de la cuál pueden establecerse relaciones con premisas etiológicas, naturales o sociales de los daños estudiados, esta dirección es comúnmente empleada en la Geografía Médica, dentro de la Nosogeografía.

También en el campo de la Salud Pública, se desarrolla una dirección encaminada a la elaboración de diseños epidemiológicos y de técnicas estadísticas para el estudio de patrones espacial es y temporales de enfermedades. La extensa literatura producida a partir de la década de los 80, evidencia la renovación del interés por este tema (Werneck $\&$ Struchiner, 1997).

Outro enfoque proveniente de la evolución de la Geografía Crítica o la nueva geografía considera el espacio como un conjunto de relaciones y de formas que se presentan como testimonio de una historia escrita por los procesos del pasado y del presente Santos (1990:138). Es naturaleza modificada por la acción humana y por tanto una construcción social.

Esta conceptualización de espacio ha sido fundamentalmente explorada por epidemiólogos, a pesar de que el arsenal teórico proviene de la Geografía (Ferreira, 1991; Gadelia, 1995). Sabroza (1991) colocaba que "el espacio socialmente organizado, integrado y profundamente desigual, no apenas posi bilita, sino determina 
Ia ocurrencia de endemias y su distribución" (Sabroza, 1991:12), en tanto Silva (1992, 1997), lo calificaba como un recurso teórico y un potente instrumento de análisis, resaltando como su más importante aporte, el brindar la visión histórico-dinámica que exige el conocimiento del proceso salud-enfermedad (Silva, 1992, 1997).

La salud de la población constituye la expresión de determinantes y condicionantes de carácter estrictamente biológicos, ambientales y sociales tanto histórico como actuales. Por otra parte en la Geografía coexiste la alta complejidad que proporcionan las relaciones de la Naturaleza, las relaciones humanas con la naturaleza y las relaciones entre los propios hombres, gestadas en una larga evolución. Así la producción social del espacio, los procesos de su configuración y funcionamiento, está influenciado por las condiciones (recursos) naturales y determinado tanto por los modos de producción precedentes como por los actuales a escala local, nacional y hoy mundial. Lo caracterizan por tanto su totalidad, historicidad y escala (Iñiguez, 1994).

La situación de salud de un espacio poblacional dado, en un momento dado, está influenciado tanto por los avatares de las formaciones económicas, de las persistencias de origen natural (clima, suelos, relieve y otras), como por la experiencia biológica de la población en contacto con diversos agentes patógenos (Dubos, 1989). Así todo espacio geográfico poblacional, portará una historia ecológica, biológica, económica, conductual, cultural, en síntesis social, que inobjetablemente ha de orientar el conocimiento del proceso salud-enfermedad, tanto como es capaz de contener y reflejar los cambios del presente, especialmente relacionados con la al ta movilidad de la población.

Diversos modelos de abordajes con propósitos explicativos o aplicados a la elaboración de políticas sanitarias, coinciden en que la salud es resultado de complejas y dinámicas interacciones entre factores, que esencialmente expresan las interacciones hombre-medio. El marco teórico que sustenta los modelos de determinantes del estado o la situación de salud de la población, evidencia fuertes nexos con el soporte teórico del espacio geográfico.

Resultan ampliamente conocidos los que definen como factores determinantes: los estilos de vida, el medio ambiente (físico y social), la biología humana y los servicios de atención de salud. Una propuesta mas reciente (Castellanos, 1992), parte de la categoría de condiciones de vida y propone su operacionalización en las dimensiones ecológica, económica, biológica y de conciencia-conducta, para identificar los perfiles diferenciales de daños a la salud en grupos poblacionales. Esta propuesta incluye las condiciones ambientales (dimensión ecológica), dentro de las condiciones de vida y considera explícitamente que los daños a la salud son resultantes tanto de la dinámica interna de estas dimensiones, como de las respuestas sociales ante procesos de cambio.

En general los modelos contemplan la complejidad de las interacciones, de lo cual se deriva la multicausalidad aceptada, pero poco aprehendida en las evaluaciones del estado de salud de diferentes poblaciones y el necesario equilibrio que ha de caracterizar la conjugación de los factores que intervienen en esta trama (Castellanos, 1991; Suárez \& Márquez, 1995).

Conocer e interpretar la diferenciacion espacial del proceso salud-enfermedad. Las limitaciones

Pudiera considerarse que los pasos esenciales en la implementación de los enfoques geográficos en los análisis sobre la desigual distribución del bienestar y la salud de las poblaciones sería conocer, conocer para interpretar, e interpretar para actuar.

Resulta frecuente que estos pasos aparezcan desarticulados, en diferentes países e incluso al interior de los mismos, tanto en el ámbito académico como sociopolítico. Especialmente en la administración en salud, el conocimiento es incompleto o deficiente y la interpretación infrecuente, las intervenciones por tanto, en el caso en que sean posibles, no favorecen la generación de alternativas o la toma de decisiones racionales y equitativas.

La primera cuestión metodológica que enfrenta el conocimiento y la interpretación del proceso salud-enfermedad es la definición de las fuentes de información que permiten decidir las unidades y escalas de observación de los procesos, como es común en diversas direcciones de investigación geográfica. Así el conocimiento responde directamente a la información, distinguiéndose aquella generada por el Sistema estadístico (secundaria), de aquella generada por investigaciones (primaria).

El Sistema de información en salud presentan diferentes niveles de generalidad y confiabilidad, identificándose limitaciones entre las cuales se destacan:

1) La escasa regularidad de la integración de los datos de la medicina privada, con la pro- 
veniente de otras instituciones gubernamentales, tales como los seguros sociales y laborales.

2) La inexistenica o incapacidad de los servicios de salud en grandes extensiones territoriales de notificar de forma sistemática y vertical la información de morbilidad.

3) La inexistencia o existencia con deficiente calidad de los registros de defunciones.

La parcialidad que aportan los subregistros se convierten en el problema inicial para el conocimiento de la desigual distribución de problemas de salud. De esta forma en ocasiones la distribución es reflejo de la calidad del sistema de información, identificándose "áreas enigmas" (Meade et al., 1988), con muy altas incidencias muchas veces explicadas por la mayor capacidad de diagnóstico, o sin casos "silencio epidemiológico" que expresa la ausencia de notificaciones y no de enfermos.

A los problemas de información se integran los de las unidades de medición de problemas de salud, en contextos espaciales caracterizados por ambientes biofísico-naturales y sicosociales relativamente homogéneos internamente y por tanto mas heterogéneos en relación con otros espacios poblacionales.

Los registros estadísticos de enfermedades y muertes están referidos a unidades territoriales que representan límites jurídicos de poder político-administrativo. La estabilidad de estas unidades es variable en los diferentes países, coexitiendo frecuentemente unidades históricas con las de delimitación reciente. En ocasiones el Sistema de Salud, define sus propias unidades en el interior de los países, considerándo exclusivamente el equilibrio numérico de las poblaciones contenidas en ellas.

Generalmente conocemos en función de la promediación, de las medias estadísticas que encubren diferencias muy sensibles entre unos espacios y otros (George, 1983:59). Mientras mas heterogéneos sean los contextos territoriales en los cuales se conoce un problema de salud, mas imprecisa resulta la interpretación de su distribución, pudiendo disminuir la efectividad de los programas de acción.

Unidades independientes de las políticoadministrativas han sido propuestas como resultado de investigaciones de la transmisión y difusión de enfermedades, generalmente infecciosas y parasitarias. Entre ellas se destacan la patobiocenosis, como áreas con una estructura biótica particular en su teoría de focos naturales (Pavlosky, 1939), o nosoáreas definidas por la elevada incidencia de una u otra entidad (Darchenkova, 1988). Encaminadas a revelar las desiguldades e inequidades en América Latina han sido utilizadas las unidades socio-es- paciales (Blanco \& Saenz, 1991), corepidemas (Breilh \& Granda, 1991), territorio-población, o espacio-población (Castellanos, 1992). Dehecho también los SILOS (Sistemas Locales de Salud), se asumen con una connotación similar (Paganini \& Chorny, 1990).

El interés por utilizar unidades más homogéneas, responde a juicio del profesor argentino J. Samaja a las exigencias de encontrar tipologías o taxonomías de "unidades genuinas" de las formaciones sociales, con toda su riqueza y dinámica reproductiva (Samaja, 1994). Es también reflejo de la necesidad estratégica de la descentralización y el incremento del significado de lo local, hecho que aparece como una nueva esperanza para la minimización de los daños o deterioros de la salud, mediante el reforzamiento de la atención primaria, el incremento de la participación comunitaria y la búsqueda de la colaboración intersectorial.

La escala de observación es otra condición en el conocimiento e interpretación de la diferenciación espacial. La escala en general responde a un nivel territorial de abordaje y aunque en asociación íntima con la unidad, no define la precisión del conocimiento. Esto es a la misma escala, se puede conocer mejor según las unidades de observación que se empleen, lo cuál no necesariamente significa conocer en una mayor fragmentación territorial.

La escala y la unidad se definen según actuaciones administrativos-operacionales o propósitos investigativos. En instituciones regionales o internacionales se emplea mas frecuentemente la unidad-escala país, mientras unidades-escalas sub-nacionales (Departamentos, Estados, Provincias o sus unidades inferiores, municipios, cantones o distritos), son del dominio de las instituciones nacionales, generalmente Ministerios de Salud, de Salud Pública, o de Salud y Ambiente, o de Universidades $u$ otros centros académicos.

Descender la escala de medición hasta unidades mas pequeñas, no necesariamente asegura el mayor poder de análisis de distribución de una enfermedad, no solo porque la unidad mas pequeña, por ejemplo el sector censal, puede contener sub-espacios desiguales, sino porque muchas veces el conocimiento y la interpretación depende de procesos, dificilmente aprehensibles en estas escalas. En este sentido se ha llamado la atención sobre la diferencia entre explicar los casos y explicar la incidencia (Rose, 1985).

Con frecuencia las instituciones nacionales y supra nacionales, emplean la agregación de unidades político-administrativa en regiones. Esta unidad taxonómica se define según múlti- 
ples criterios, dependiendo del problema de salud de que se trate, resultándo en la actualidad mas frecuentes que los Programas de Control definan estratos que agregan países o su unidad política inferior, con similar situación epidemiológica. Una propuesta prometedora, aún poco explorada parece ser la de conocer la situación de salud según estratos que integren territorios - poblaciones relativamente homogéneos según condiciones de vida (Castellanos, 1992).

Resulta total mente aceptado que enormes desigualdades e inequidades en salud, se despliegan en el contexto Latinoamericano, no obstante desconocemos en cuanto se enmascara la criticidad de estas realidades, en tanto son constatadas como promediación de la frecuencia de uno u otro evento nosológico en una unidad que contiene poblaciones con condiciones ambientales y de vida radicalmente desiguales. No deja de ser paradógico, además de contradictorio con el marco teórico del proceso salud-enfermedad, que se acepte la profunda división de la Sociedad en clases, o la concentración de población en ciudades y regiones metropolitanas y no se cuente con información de salud para estas u otras agregaciones humanas reales de forma sistemática.

Particularmente agudo resulta este hecho en las informaciones sobre enfermedades infecciosas de transmisión hídrica, prevenibles por vacunas o sustentadas en la desnutrición, con ocurrencia intensa en determinados grupos y espacios poblacionales.

Si aceptáramos que la salud es expresión del bienestar físico, mental y social y no solo la ausencia de enfermedad (OMS, 1964), como presupuesto teórico, resulta evidente que lo que comúnmente se mide por el Sistema de Salud es la frecuencia de la enfermedad, en tanto efecto negativo e indirecto del bienestar físico y parcialmente del mental.

Mientras el bienestar social, expresión de las condiciones materiales de vida (objetivosubjetivo) y de la percepción que los grupos poblacionales tienen de sus vidas (subjetivoobjetiva), es medida por otras estructuras fuera del Sistema de Información en Salud, y que en teoría pero muy débilmente en la práctica, pertenecen al denominado Sector Salud.

Este otro hecho ampliamente debatido, tambien limita el conocimiento de la situación de salud en los diferentes espacios-poblacionales, considerando que las realidades humanas son tanto realidades espirituales como materiales, pues la imagen que tienen los hombres de su bienestar y de su salud son tan im- portantes como la masa de bienes de las que disponen (Smith, 1980).

Por último cabe una reflexión indispensable acerca del propagado interés por los Sistemas de información geográfica en salud en las últimas dos décadas, o lo que se ha denominado de forma mas general el Geoprocesamiento de información en salud, que incorpora otras fuentes de información relativamente novedosas, como las imagénes satelitarias.

Los Sistemas de Información Geográfica en Salud (SIGSA) o en epidemiología (SIG-epi) se califican como una herramienta de apoyo en el análisis de situación de salud, en la vigilancia, planeación y evaluación de intervenciones en el Sector Salud (Castillo-Salgado, 1996). La ventaja de manejar grandes volúmenes de información georeferenciadas con rapidez, promueve su amplia aceptación.

Quizás el efecto mas significatico es el incremento vertiginosos de la utilización de los mapas. La vuelta a los mapas, abandonados y secundarios en el quehacer de los salubristas y el reconocimiento actual de su utilidad, es resultado directo del acelerado desarrollo de la informática y de uno de sus instrumentos privilegiados, las computadoras personales.

La distribución geográfica de problemas de salud o de componentes del Sistema adquieren tal importancia que se estima que aproximadamente el $80 \%$ de las necesidades de información de quienes toman decisiones o definen políticas en los gobiernos locales está relacionado con una ubicación geográfica (Williams, 1987).

Sin deconsiderar la utilidad potencial de estas técnicas, debe considerarse que la cartografía (automatizada o nó), mejora el conocimiento o la interpretación de la distribución de daños a la salud, en dependencia de la calidad de los Sistemas de Información. Estos son dependientes de la accesibilidad física y social a los servicios de salud, la cobertura y calidad de estos servicios, así como de los Sistemas Estadísticos resultantes de los fluxos verticales y horizontales de información. Por tanto los SIGSA o los mapas, no eliminan sino reproducen las deficiencias de la información en salud.

Los mapas pueden también convertirse en un recurso peligroso, al conseguir de una forma atractiva reducir la preocupación por las limitaciones de la calidad de información y de las unidades de medición, además de incorporar otras nuevas deficiencias derivadas del desconocimiento o utilización incorreta de métodos de representación cartográfica.

Sería conveniente suscitar reflexiones acerca de las potencialidades que las técnicas de 
Geoprocesamiento brindan para la reformulación del proceso de medición de eventos y procesos determinantes de daños a la salud y en la definición de los espacios de su producción. Observar tanto sus fortalezas como sus debilidades, para determinar modelos y patrones de distribución a partir de unidades político administrativas $u$ otras unidades alternativas definidas al efecto y la forma en que estas técnicas varían la concepción clásica de escala de observación. Atención especial tendrían las reflexiones acerca de la importancia de los SIGSA y del proceso de su "alimentación"como condición indispensable para su funcionamiento.

Incorproración del espacio geográfico en el conocimiento de la situación de salud en América Latina

Los retos de la descentralización y la ingente necesidad de identificar prioridades, exigen de un cambio de enfoque en el conocimiento de la espacialidad del proceso salud-enfermedad, en el quehacer epidemiológico y particularmente en la vigilancia en salud. Privilegiar el potente arsenal que brinda la Geografía y la conceptualización del espacio geográfico pudiera ser una vía para aprehender en cada país los eventos de salud, acorde a los marcos contrastantes en los cuales transcurre la vida de la población.

Entre los procesos que más han afectado las condiciones de vida y la salud de la población en los últimos decenios, se destacan las desigualdades e inequidades sociales, el intenso proceso de urbanización, los cambios en la composición de la fuerza laboral, en la estructura de edad de la población, en el nivel educativo así como la organización de los servicios públicos y especialmente el papel del gobierno ante ellos (OPS, 1994).

En la década de los 80 la mayoría de los países latinoamericanos experimentaron una aguda crisis económica que obligó a la aplicación de ajustes estructurales en los modelos de desarrollo. De ello resultó una pérdida de capacidad de los estados nacionales para invertir en áreas sociales, especialmente en los servicios de bienestar y salud de la población, desestabilizados aún mas, con el incremento de la privatización y algunos resultados desfavorables de la descentralización.

También en esta década, la Organización Panamericana de la Salud, propone analizar y vigilar la salud, según condiciones de vida, representando esto tanto un acercamiento del marco conceptual a la observación del proceso salud-enfermedad, como una posilbilidad real de revelar con claridad las desigualdades y especialmente las inequidades (OPS, 1995).

Si el espacio geográfico se considera como mediador para la distribución de los daños en un espacio social concreto (Breilh \& Granda, 1990), también las condiciones de vida se consideran mediadoras de las diferencias de los problemas de salud y constituyen la expresión concreta de la forma en que cada grupo de población participa en el proceso general de reproducción de la sociedad, en un lugar, y un momento histórico determinado (Castellanos, 1991, 1992).

Se integra a este hecho y en alguna medida lo condicionan la ingente necesidad de procurar de forma armónica y articulada el mejoramiento del bienestar de las poblaciones, mas allá de los intentos por mejorar la atención médica, en la dirección hacia una práctica de la salud pública dirigida a promover salud y no preferencialmente a cuidar la enfermedad (Sabroza, 1994).

Paral elamente la evolución de la Medicina Social y en general a los enfoques que rompen la unidireccionalidad de la medicina biologicista, refuerzan la utilidad del conocimiento de la distribución de los perfiles diferenciales de daños, por que en las unidades geográficas es donde operan los procesos determinanes (condiciones de vida), se expresan los problemas de salud-enfermedad y se desarrollan las acciones para el mejoramiento de la salud y el bienestar.

Se ha planteado que las mayores potencialidades de incorporación de la conceptualización del espacio geográfico parecen identificarse en el campo de la Salud Pública. Caracterizada por un nivel de análisis poblacional, obligada a ser interdisciplinar en la investigación e intersectorial en la acción, es capaz de articular tanto las acciones gubernamentales, con las de las comunidades educadas y organizadas, como los servicios para el diagnóstico y tratamiento de enfermedades o discapacidades con los que procuran el mejoramiento ambiental físico y social (Rojas-Ochoa, 1993).

El tema de la salud o la enfermedad está generalmente ausente en el quehacer de los geógrafos latinoamericanos y es solo esporádicamente tratado en eventos y publicaciones geográficas.

Un proyecto interinstitucional para el desarrollo de la Geografía Médica en Venezuela fue propuesto por el Departamento de Geografía de la Universidad Central (UCV) (González, 1992). Entre otras razones, el temprano fallecimiento de su principal promotor, el profesor 
Enrique González Boscán, paralizaron este empeño.

Numerosas acciones han sido desarrolladas en México, sin que se pueda calificar de consolidada esta dirección ni en el ámbito académico ni institucional, entre ellas se destacan publicaciones tales como "Fundamentos de la Geografía Médica (Sáenz de la Calzada, 1956), Técnicas en Geografía Médica (Aguilar, 1989), - la Memoria de la reunión de trabajo sobre Geografía de la Salud, realizada en Chiapas (Dickinson, 1981).

Recientemente Panamá ha publicado su tercer Atlas de Salud. Estas publicaciones promovidas por geógrafos destacados del país ( $L$. Herrera y L. Castro) y el Ministerio de Salud, integrando un gran número de instituciones, a pesar de que la Geografía Médica o de la Salud es una dirección prácticamente desconocida en el país.

Quizás el caso de Cuba sea excepcional por cuanto existen grupos de investigación con larga trayectoria en esta temática en el Centro de Estudios de Salud y Bienestar Humanos de la Universidad de la Habana y el Instituto de Geografía Tropical del Ministerio de Ciencia, Tecnología y Medio Ambiente. En los últimos años se ha establecido la incorporación progresiva de geógrafos en diferentes instancias del Ministerio de Salud Pública, en el nivel central y provincial, fundamental mente dedicados a la Vigilancia en Salud y el desarrollo de Sistemas de Información Geográfica en Salud.

Sin pretender profundizar las causas, resulta fundamental reconocer que la formación de pregrado del profesional de la Geografía, está débilmente dotada del arsenal que propicie la acción transdisciplinar, con otras Ciencias Humanas, especialmente en Biología, Epidemiología, Sociología, Psicología, Antropología Física y Social, entre otras, lo cuál no significa que su inclusión en el curriculum de estudio resolvería tal situación.

Por otra parte la carencia de centros o instituciones geográficas que investiguen sobre estos temas, constituye quizás el principal freno para su potenciación. De lo anterior se desprende la consecuente carencia de centros de formación pos-graduada en esta especialización. No menos importante es el desconocimiento o la falta de reconocimiento de la potencialidad de este profesional en instituciones de salud.

Un hecho cierto es que algunos geógrafos latinoamericanos han intentado pero no han logrado consolidar a la Geografía Médica o de la Salud, en la historia de la formación académica e inserción institucional de este profesional, aunque continúa siendo una opción.
Mientras, toda una experiencia se concreta y consolida desde la Salud Pública, especialmente desde la Epidemiología y la Medicina Social en el orden teórico y metodológico, en relación con la distribución de los dañs a la salud. Los procesos que han acompañado la renovación de las estrategias, condicionadas por la aguda crisis en la situación de salud de grandes grupos poblacionales, ha provocado el resurgimiento de intereses "aparentemente olvidados" por incorporar los conocimientos geográficos y especialmente de los emanadaos de la Geografía Crítica, como una opción en la interpretación del bienestar, la salud y sus diferenciales.

Se identifican hoy en Latinoamérica grupos y centros docentes e investigativos, que articulan e integran, tanto la base conceptual del espacio geográfico, la construcción y organización de los espacios geográficos, como las técnicas más actuales de la investigación geográfica, con resultados destacados especialmente en el campo de las enfermedades infecciosas y parasitarias, tales como malaria, oncocercosis, leishmaniasis, Chagas, lepra y el cólera entre otras.

Cabe resaltar entre ellos, el Departamento de Grandes Endemias de la Escuela Nacional de Salud Pública de la Fundación Oswaldo Cruz y el de la Facultad de Medicina de la Universidad de Bahía, ambos en Brasil, el Instituto Juan César García y el Centro de Estudios y Asesoría en Salud, la Universidad Autónoma de Xochimilco y el Centro de Investigaciones Avanzadas Regional Mérida, ambos en México. Aunque la producción es muy superior a la de los geógrafos aislados que desarrollan estos enfoques, de forma similar se identifica la inconstancia y la desarticulación de estas producciones.

En el quehacer geográfico de muchos países latinoamericanos, encontramos una vasta producción histórica y actual de conocimientos sobre la diferenciación espacial de fenómenos y procesos que sustentan las desigualdades e iniquidades en situación de salud y bienestar de las poblaciones. Como promover el interés de los geográfos o de los profesionales de salud por articular o potenciar tan evidente vinculación?

\section{A modo de reflexiones finales}

La forma en que transita el "desarrollo en el subdesarrollo", es la principal causa de la distribución desigual de los problemas de salud. Ello sustenta tanto la permanencia de los condicionantes del ambiente natural, como las pro- 
fundas desigualdades e iniquidades sociales determinantes de la vida, incluyendo la de las prácticas de intervención en salud.

La progresiva transformación del medio o del ambiente en medio científico-técnico-informacional, (Santos, 1985, 1994), acrecienta poderosamente las disarmonías en las relaciones hombre-medio, y entre los propios hombres y propician el incremento de las injusticias sociales. Entender estos hechos como rotundos, no implica aceptarlos como definitivos. Los deterioros del ambiente (que asumen progresivamente el principal papel protagónico), de las condiciones de vida, de la salud y del bienestar fuerzan la convergencia profesional de objetivos y enfoques.

La interpretación de la espacialidad del proceso salud-enfermedad exige de la integración del conocimiento geográfico y se nutre de su fragmentación, es además un quehacer obligatoriamente transdisciplinar. La proximidad y complementariedad de los marcos conceptuales entre la geografía y la salud son evidentes, mucho más evidente para los profesionales de la salud que para los de la geografía, la posibilidad de articular fundamentos metodológicos y técnicas de investigación está identificada, aunque no suficientemente explorada.

Las investigaciones geográficas utilizan escasamente los datos indirectos de salud y los actores encargados de interpretar y revertir los deterioros desiguales en salud, también escasamente se nutren de los conocimientos emanados de los estudios geográficos.

Asumir el espacio como sistema de objetos y acciones (Santos, 1996), como herencia, como construcción social, posibilita interpretar y actuar de acuerdo a donde y como las colectividades viven, es una opción que enfrenta no pocos problemas de orden teórico y operacional, entre los cuales se destacan la necesidad de elaborar nuevos conceptos, de definir espacios alternativos de medición de procesos y la construcción de modelos particulares de interpretación para determinados contextos y enfermedades y hasta el manejo de los espacios y números pequeños.

Mas que reafirmar el reconocimiento histórico de la utilidad de los enfoques geográficos para la comprensión del proceso salud-enfermedad, mas que intentar potenciar el desarrollo de una u otra disciplina, se precisa fomentar el diálogo entre geógrafos y profesionales de la salud e identificar las perspectivas de su aplicación y sus potencialidades para revelar las desigual distribución de daños a la salud y el bienestar humano, en el contexto latinoamericano.
Muchos de estos problemas son comunes a otros abordajes teórico-metodológi cos que desarrollan técnicas cuantitativas o tecnologías de geoprocesamiento de información en salud. Avanzar por diversos caminos no es erróneo, lo imperdonable sería que estos fueran divergentes. Las potencialidades de instrumentación de una u otra alternativa en diversos países de la región, dependerá de contextos coyunturales, tanto como del surgimiento o identificación de líderes.

La pobreza, la miseria, el hambre y la desnutrición, la insalubridad, la desesperanza, la tristeza, la violencia, la contaminación del aire de las aguas, las tierras y tanto otros males definen espacios que se construyen y reconfiguran permanentemente, también los procesos y estados contrarios definen espacios particulares. En unos y en otros, heredados o emergentes acechan o dramáticamente se despliegan las enfermedades y las muertes. Identificar y revelar estos espacios humanos es una posibilidad de mejorar la efectividad y la equidad de las acciones en salud. Es una responsabilidad y una urgencia. 


\section{Referencias}

AGUILAR, L., 1989. Técnicas en Geografía Médica. México: Limusa.

BLANCO, J. \& SÃENZ, Z. O., 1991. Condiciones deVida y Salud en el Ámbito Urbano. La Habana: Facultad de Salud Pública.

BREILH, A.; CAM PAÑA, A. \& GRANDA, E., 1988. Geografía delas Condiciones deSalud-Enfermedad en el Ecuador. Quito: CEAS.

BREILH, J. \& GRANDA, E.,1990. Deterioro de la Vida. Quito: Corporación Editora Nacional.

CASTELLANOS, P. L., 1991. Sistemas Nacionales de Vigilancia dela Situación de Salud según Condiciones de Vida y del Impacto de las Acciones de Salud y Bienestar. Washington: OPS/OMS.

CASTELLANOS, P. L., 1992. Perfiles de salud y condiciones de vida: Una propuesta operativa para el estudio de las inequidades en salud en América Latina. Granada: Anales del I Congreso I beroamericano deEpidemiología, pp. 7-8.

CASTILLO-SALGADO, C., 1996. Uso de los sistemas de información geográfica en epidemiología. Boletín Epidemiológico de la Organización Panamericana dela Salud, 17:1-6.

CLAVAL, P., 1987. Geografía Humana y Económica Contemporánea. Madrid: Ediciones Akal.

DARCHENKOVA, N. P., 1986. Significado, Tareas, Desarrollo y Objeto de la Geografía Médica. Disertación de Maestría, La Habana: Facultad de Geografía, Universidad de La Habana.

DICKINSON, F., 1981. I Reunión deTrabajo sobreGeografía de la Salud. Chiapas: Centro de Investigaciones Ecológicas del Sureste.(mimeo.)

DUBOS, R., 1989. El Hombreen Adaptación. México: Fondo de Cultura Económica.

FERREIRA, M. U., 1991. Epidemiologia e geografia. O complexo patogênico de Max. Sorre. Cadernos de SaúdePública, 7:301-309.

GADELIA, P. E., 1995. História das Doenças. Pontos de Encontros eDispersões. Tese de Doutorado, vol. II, Rio de Janeiro: Escola Nacional de Saúde Pública, Fundação Oswaldo Cruz.

GEORGE, P. G., 1983. Geografía de las Desigual dades. Barcelona: Oikos-Tau.

GONZALEZ, E., 1992. Breve Reseña Descriptiva del Proyecto "Geografía Médica en Venezuela". Caracas: Escuela Nacional de Geografía, Universidad Central de Venezuela.(mimeo.)

IÑIGUEZ, L., 1994. Espacio geográfico y salud de la población. Memorias del 60 Congreso Latinoamericano y 80 Congreso Mundial de Medicina Social. México: Alames (Asociación Latinoamericana de Medicina Social).

JENICEK, M. \& CLÉROUX, R., 1987. Epidemiología. Principios. Técnicas. Aplicaciones. Barcelona: Ediciones Científicas y Técnicas.

LILIENFIELD, A. M. \& LILIENFIELD, D. E., 1986. Fundamentos de Epidemiología. México: Sistemas Técnicos de Edición.

MAUSNER, J. \& KRAMER, S., 1985. Epidemiología. Un Texto Introductorio. Philadelphia: Saunders Company.

MAY, J. M., 1950. Medical geography. Its methods and objectives. Geographical Review, 40:9-41.
McGLASHAN, N. D., 1983. Aspectos Geográficos de la Salud. London: Academic Press.

MEADE, M.; FORIN, J. \& GESLER, W., 1988. Medical Geography. New York: The Guilford Press.

MOLLISON, D., 1996. Epidemic Models: Their Structureand Relation to Data. Cambridge: Cambridge University Press.

OMS (Organización Mundial de la Salud), 1964. Constitución de Ia OMS. Documentos Básicos. XV Asamblea. Ginebra: OMS.

OPS (Organización Panamericana de la Salud), 1994 Las Condiciones de Salud en las Américas. Washington: OPS/OMS.

OPS (Organización Panamericana de la Salud), 1996. Inequidades en salud y la región de las Américas. Boletín Epidemiológico de la Organización Panamericana dela Salud, 17:1-9.

PAGANINI, L. L. \& CHORNY, A. H., 1990. Los sistemas locales de salud: desafíos para la década de los noventa. Boletín de la Organización Panamericana dela Salud, 109:436-450.

PAVLOSKY, E. N., 1966. Natural Nidality of Transmisible Diseases. Illinois: University of Illinois Press.

PYLE, G., 1977. Introduction: foundations to medical geography. Economic Geography, 52:95-123.

RATZEL, F., 1948. Antropogeografía. Tomo I. La Habana: Facultad de Filosofía y Letras, Universidad de La Habana.

ROJAS-OCHOA, F., 1993. Al gunos Conceptos y Prácticas sobre Salud Pública, Promoción de Salud y Educación para la Salud. La Habana: Grupo Interdisciplinario de Estudios en Salud.

ROSE, G., 1985. Individuos enfermos y poblaciones enfermas. Journal of Epidemiology, 14:32-38.

ROU QUAYROL, M. Z., 1994. Epidemi ol ogia e Saúde. São Paulo: Editora M édica e Científica.

SABROZA, P. C., 1991. Espaço e Produção de Endemias. Caracas: Segundo Taller de Alames (Asociación Latinoamericana de Medicina Social). (mimeo.)

SABROZA, P. C., 1994. Saúde Pública: procurando os limites da crise. I Conferência Panamericana de Educação em Saúde Pública. Resumos. Rio de Janeiro: Escola Nacional de Saúde Pública, Fundação Oswaldo Cruz.

SÃENZ DE LA CALZADA, C., 1956. Los fundamentos de la geografía médica. Boletín de la Sociedad Mexicana de Geografía y Estadística. LXXXI:I .

SAMAJA, J., 1994. Vigilancia Epidemiológica de los Ambientes en que se Desarrollan los Procesos de Reproducción Social. Memorias del 6o Congreso Latinoamericano y 80 Congreso Mundial de Medicina Social. México: Alames (Asociación Latinoamericana de Medicina Social).

SANTOS, M., 1990. Por una Geografía Nueva. Madrid: Espasa Calpe.

SANTOS, M., 1994. A Questão do Meio Ambiente: De safios para uma Perspectiva Interdisciplinar. São Paulo: Edusp.

SANTOS, M., 1996. A Natureza do Espaço. São Paulo: Hucitec.

SILVA, L. J., 1992. Organização do espaço e doença. In: Epidemiologia I. Textos de Apoio (J. R. Carva- 
Iheiro, org.), vol. 1, 2a ed., pp. 59-85. Rio de Janeiro: Escola Nacional de Saúde Pública, Fundação Oswaldo Cruz.

SILVA, L. J., 1997. O conceito de espaço na epidemiologia das doenças infecciosas. Cadernos de SaúdePública, 13:585-593.

SMITH, D., 1980. Geografía Humana. Barcelona: Oikos-tau.

SORRE, M., 1955. Fundamentos Biológicos de la Geografía Humana. Barcelona: Juventud.

SOUNIS, E., 1985. Epidemiologia Geral. Curitiba: Universidade Federal do Paraná.

SUAREZ, J. \& MARQUEZ, M., 1995. Los proyectos locales de promoción de salud. Nuevos escenarios para el desarrollo de la Salud Pública. Serie Desarrollo dela Representación OPS/OMS en Cuba.

VERHASSELT, Y., 1993. Potentialities of Geography of Health. Archives of Public Health, 51:481-486.

VIDAL DE LA BLACHE, P., 1922. Principes de GéographieHumaine Paris: A. Colin.

WERNECK, G. L. \& STRUCHINER, C. J., 1997. Estudos de agregados de doença no espaço-tempo: conceitos, técnicas e desafios. Cadernos deSaúde Pública, 13:611-624.

WILLIAMS, R. E., 1987. Selling a geographycal system to goverment policy makers. Boletín Epidemiológico de la Organización Panamericana de la Salud, 17:1. 